\title{
Changes in soil chemical and biological properties as influenced by organic nutrient management practices under rainfed condition
}

N. J. Gowthamchand, Ganapathi and T. M. Soumya

Received : 18.12.2019; Revised : 01.05.2020; Accepted : 18.05.2020

MEMBERS OF RESEARCH FORUM:

Corresponding author : Ganapathi, Main Agricultural and Horticultural Research Station, Iruvakki, Anandapuram, Sagara, Shivamogga (Karnataka) India Email: fsiruvakki@gmail.com
Co-authors :

${ }^{1}$ N.J. Gowthamchand, Department of Soil Science, Punjab Agricultural University, Ludhiana

(Punjab) India

${ }^{2}$ T.M. Soumya, Department of Agronomy, ZAHRS, UAHS, Shivamogga (Karnataka) India

\section{Summary}

A field experiment was conducted to study the effect of organic manures and fermented liquid organic formulations on growth, on soil chemical and biological properties under rainfed condition at Organic Farming Research Centre, Zonal Agricultural and Horticultural Research Station (ZAHRS), Navile, Shivamogga, during Kharif 2018-19. The experiment was laid out in Randomized Block Design with ten treatments comprising of different bulky organic manures (FYM and Vermicompost) and fermented liquid organic formulations (Beejamrutha, Jeevamruta, Panchagavya and Cow urine) were replicated thrice, French bean variety Arka sharath taken as a test crop. Experimental results revealed that, among various treatments, at harvest significantly higher soil $\mathrm{pH}(6.10)$, electrical conductivity $\left(0.092 \mathrm{dS} \mathrm{m}^{-1}\right)$ and organic carbon $\left(4.60 \mathrm{~g} \mathrm{~kg}^{-1}\right)$ status were observerd with application of Beejamrutha (seed treatment) + Jeevamrutha (soil application@ $500 \mathrm{~L} \mathrm{ha}^{-1}$ ) + $100 \%$ RDN through vermicompost + Foliar spray of Panchagavya @ 3\% ( $\left.\mathrm{T}_{6}\right)$ over treatment which received Beejamrutha (seed treatment) + Jeevamrutha (soil application @ 500 L ha-1). The available nitrogen $\left(296.80 \mathrm{~kg} \mathrm{ha}^{-1}\right)$, phosphorus ( $\left.98.61 \mathrm{~kg} \mathrm{ha}^{-1}\right)$, potassium $\left(173.18 \mathrm{~kg} \mathrm{ha}^{-1}\right)$, exchangeable $\mathrm{Ca}, \mathrm{Mg}$, available sulphur and DTPA-extracted micro nutrients ( $\mathrm{Zn}, \mathrm{Cu}, \mathrm{Fe}$ and $\mathrm{Mn}$ ) status were significantly higher at harvest stage in treatment which received Beejamrutha (seed treatment) + Jeevamrutha (soil application@ @ $\left.500 \mathrm{~L} \mathrm{ha}^{-1}\right)+100 \% \mathrm{RDN}$ through vermicompost $\left(\mathrm{T}_{3}\right)$. Dehydrogenase, acid phosphatase and urease activities in soil at different growth stages of french bean were significantly increased in all treatments due to application of organic sources over control which received Beejamrutha (seed treatment) + Jeevamrutha (soil application@ $500 \mathrm{~L} \mathrm{ha}^{-1}$ ).

Key words : Vermicompost, Beejamrutha, Panchagavya, Jeevamruta, FYM

How to cite this article : Gowthamchand, N.J., Ganapathi and Soumya, T.M. (2020). Changes in soil chemical and biological properties as influenced by organic nutrient management practices under rainfed condition. Asian J. Soil Sci., 15 (1) : 1-10 : DOI : 10.15740/HAS/AJSS/15.1/1-10. Copyright@2020: Hind Agri-Horticultural Society. 